\title{
Total Laparoscopic ALPPS: Extended Right Hepatectomy for Metastatic Liver Tumor
}

\author{
Nuengruetai Orannapalai, Rawisak Chanwat, Chairat Bunchaliew, Rapheephat Tanompetsanga
}

\author{
Corresponding author: \\ Nuengruetai Orannapalai, MD \\ Hepato-Pancreato-Biliary Surgery Unit \\ Department of Surgery \\ National Cancer Institute \\ 268/1 Rama VI Road, Rajthevi \\ Bangkok 10400 Thailand. \\ Tel: +6623547025 \\ Fax: +6623547025 \\ E-mail: honook@gmail.com
}

Hepato-Pancreato-Biliary Surgery Unit, Department of Surgery, National Cancer Institute Bangkok, Thailand

\section{ABSTRACT}

Background: Hepatectomy is the potentially curative treatment for liver tumors. The most critical postoperative complication of extensive liver resection is post-hepatectomy liver failure (PHLF) due to insufficient future liver remnant (FLR). The ALPPS (Associating Liver Partition and Portal vein Ligation for Staged hepatectomy) effectively increases the resectability of inoperable liver tumors by achieving a rapid and an effective hypertrophy of the FLR, which lowers postoperative liver failure risk. However, this technique still carries on with high morbidity and mortality rate.

Aim: To present the first case of total laparoscopic ALPPS for extended right hepatectomy which is shown in VDO resources. Our institute proposed this technique as a valid option aim to improve the outcomes of ALPPS procedure by careful patient selection.

Technique: Total laparoscopic ALPPS involves two stages. The first stage consisted in the ligation of the right portal branch and the partition of the liver. The second stage was performed on day 14 after the first stage which the patient has already confirmed the sufficient liver remnant volume and function by the MRI upper abdomen and primovist. The de-portalized liver was removed by transected the right hepatic artery (RHA), the biliary duct and the right and middle hepatic vein (RHV, MHV).

Conclusions: The ALPPS technique is a novel therapeutic method for inoperable liver tumors by conventional methods. By careful patient selection and technical adjustment to the particular conditions of each case, better outcomes have been achieved.

Key words: laparoscopic ALPPS, metastatic lymphoma of stomach

\section{INTRODUCTION}

The goals of the malignant liver surgery aim to obtain RO resection and sufficient FLR with functional reserve to avoid PHLF (1). Resection of a large liver tumor may result in an excessive removal of liver parenchyma leading to PHLF and associated complication (2). There are two effective methods to increase the volume of the FLR: portal vein ligation (PVL)/ percutaneous portal vein embolization (PVE) and two stage hepatectomy. These strategies, however, carry a considerable failure rate because a significant proportion of patients eventually drop out from subsequent curative resection due to tumor progression in the waiting interval between the two stages, or because of failure of the FLR to adequately grow. ALPPS not only allows to increase resectability of 
liver tumor in patients with borderline or inadequate FLR that would not be possible with conventional techniques, but also enables surgeons to proceed with multi-stage resections within a short time interval before a substantial tumor progression (3). Many surgeons have rapidly adopted ALPPS as it has the advantages of inducing rapid liver hypertrophy and completion of tumor resection rate for the second staged operation (4). The main criticism of ALPPS is its high morbidity and mortality rate. Thus ALPPS is not accepted by some surgeons who believe portal vein embolization or portal vein ligation to be much safer than ALPPS (5). In our institute already started up for ALPPS but shift to laparoscopic ALPPS instead of conventional ALPPS, aiming to decrease the perioperative morbidity and mortality rates, to improve postoperative long-term survival and to improve the completion of tumor resection of the second stage operation. This article aims to review our technique which is shown in VDO resources.

\section{CASE REPORT}

The patient is female 55 year old. She was diagnosed Non-Hodgkin's Lymphoma of stomach. She received chemotherapy for 6 months then subtotal gastrectomy was performed 1 year ago. During surveillance disease, we found solitary liver mass which involves right portal pedicle, RHV and MHV that also near to the hilar region as shown in Fig 1. In aspect of preoperative evaluations in non-cirrhotic patient include Child-Pugh score $=A$, ICG-R15 $=3$ that represent for allowance hepatic resection $=78 \%$ as shown in Fig. 1. We used liver volumetry program to design the operative planning. Due to tumor location as shown in Fig. 1. We had designed 2 possible operations that obtain negative surgical margin for this patient as shown in Table 1. Our plan is total laparoscopic ALPPS for extended right hepatectomy with MHV transaction that also reduce chance of PHLF with sufficient FLR and obtain negative surgical margin.

In the first stage laparoscopic ALPPS, we created totally 5 ports incision which each 2 ports for surgeon

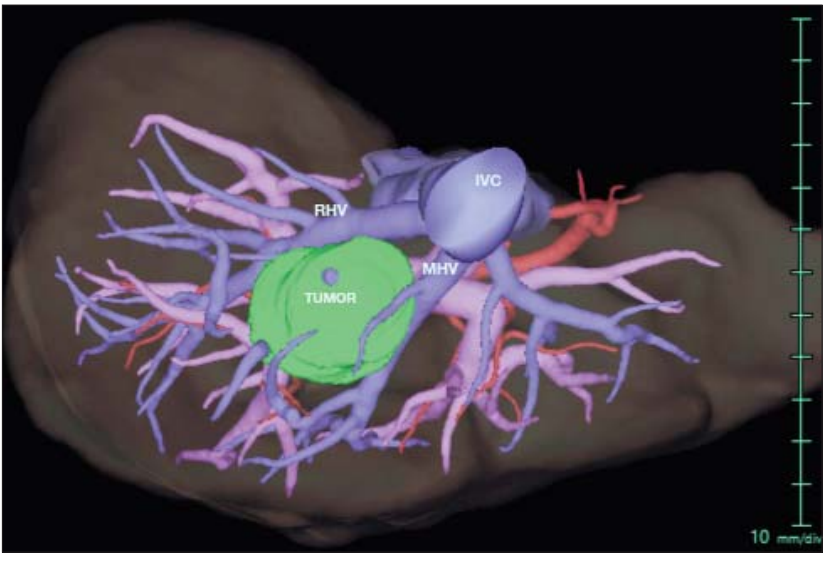

Figure 1 - Tumor location, ICG R15 and AHR

and assistant surgeon. We set up our patient in French position with reversed trendelenburg. After falciform ligament transection was done with bipolar vessel sealing. The suprahepatic inferior vena cava ligament was also dissected as the same fashion. We found some adhesion from previous subtotal gastrectomy. We start the procedure with ligation of right branch of portal vein (RPV) by intrafascial dissection after cholecystectomy. By the advantage of laparoscopic hepatectomy, the caudal view approach for individually encircled under direct visualization of the RPV and the RHA were performed and followed by tagged the RHA with Prolene 3-0. The demarcation line after ligation of the RPV was shown. The ICG fluorescence imaging was used to enhance the demarcation line. After portal ligation, the next step is liver partition. We used laparoscopic intraoperative ultrasound (IOUS) to see the MHV and tumor margin. Due to we plan for extended right hepatectomy, the transaction plain was shifted to the left from MHV about $1 \mathrm{~cm}$. Intracorporial Pringle's maneuver was applied for inflow control part before liver partition. We use Pringle's maneuver fashion as 15 minutes clamp alternate with 5 minutes release as liberal. At the liver surface about $1-2 \mathrm{~cm}$, we used ultrasonic scalpel device under traction-counter traction technique as routinely. At the deeper part of liver, we selected the CUSA for liver partition. The transaction was dept down to the hilar plate. The goal in the first

Table 1 - Preoperative evaluation by volumetry

\begin{tabular}{ll}
\hline Liver volumetry: right trisectionectomy & Liver volumetry: extended right hepatectomy \\
\hline Tumor volume $=20.5489$ & Tumor volume $=20.5489$ \\
\hline Total liver volume $=1150.6230$ & Total liver volume $=1150.6230$ \\
\hline Total functional Liver Volume $=1130.0741$ & Total functional Liver Volume $=1130.0741$ \\
\hline Remaining Liver Volume $=162.5538$ & Remaining Liver Volume $=270.5904$ \\
\hline $\mathrm{F} L \mathrm{R}=14.4 \%$ & $\mathrm{FLR}=23.9 \%$ \\
\hline
\end{tabular}


stage is redistributed total portal blood flow to the FLR. All intrahepatic venous branches were dissected meticulously and clipped until we found MHV that was encircled and tagged with Prolene 3-0. Before end of operation, we tagged the hepatoduodenal ligament, the RHA, and the MHV. We preserved the MHV in the first stage to avoid congestion effect of the deportalized liver which reduce perioperative complications. We used anterior approach of transaction to keep virgin and minimize adhesion formation in the area surround the hilar, caudate and IVC to avoid iatrogenic injury in the second stage. Notice that the left lobe liver was significantly intraoperatively oedema. However, it is not represent for the function of FLR. We selected interceed material to place in transaction plain to avoid perihepatic adhesions. We left negative pressure drainage (Jackson-Pratt drain) after complete the first stage.

We sent the MRI upper abdomen with Primovist at day 10 and found the mature functional hypertrophy and adequate volume of the remnant liver. We set up the second stage laparoscopic ALPPS at day 14 after the first operation to promote the maximum chance of the liver remnant hypertrophy. Major of the operative field was loose adhesion that can easily lysis with bipolar vessel sealing and the CUSA at the transaction plain. The RHA that was already tagged was transected in this stage. We used vascular staple for MHV transaction. The right lobe liver was full mobilization. Caudate lobe transaction was done. The RPV was also transected with vascular staple. The posterior part of the deportalized liver was transected. The RHV was transected with vascular staple. The right bile duct was clearly transected for the last. The specimen was removed. Jackson-Pratt drain was placed after complete the second stage.

\section{RESULTS}

When we designed to the possible operations of this patient, we found that the liver tumor was very closed to surgical margin. We need the procedure which can offer rapid boost up remnant liver volume to avoid drop out chance during waiting interval. Laparoscopic ALPPS for extended right hepatectomy was performed. The first stage's operative time is total 200 minutes without any complication. Estimate blood loss was $50 \mathrm{~mL}$. The patient was discharged 3 days later with drain. The MRI upper abdomen with Primovist was done at day 10 after the first stage of operation. The liver remnant was promoted from 24 to 36 percent with mature hypertrophic function. The second stage's operative time is total 180 minutes without any complication.
Estimate blood loss was $20 \mathrm{~mL}$. The patient was discharged 3 days later with drain. The pathological examination was $8 \mathrm{~cm}$ in size of metastatic poorly differentiated carcinoma, compatible with metastatic lymphoma with all free resected margin. The drainage was completely removed on out patient follow up date.

\section{DISCUSSION}

There are several literatures and studies which have demonstrated that laparoscopic hepatectomy has several advantages over open technique; reducing the rate of complication and hospitalization. The high risk of morbidity and mortality are the major criticism received by the ALPPS procedure. Laparoscopic ALLPS have started to be proposed as a valid option to improve outcomes. Our institute proposed this patient here, we proved the feasibility of accomplishing total laparoscopically ALPPS. No intraoperative or postoperative complications were diagnosed.

Importantly, these technique can safe the outcome that can make a completion of specimen before tumor progression with safe oncological margin. Furthermore, the PVL/ PVE of RPV in this patient could might be loss to perform liver tumor resection, because of failure of the FLR to adequately liver remnant grow. Larger series will define out for long term outcome and related complications if we have performed high volume of ALPPS in laparoscopically in the future.

However, there are not several situations in which we have to decide the ALPPS procedure.

\section{Disclosure}

The authors declare no conflicts of interest.

\section{REFERENCES}

1. Wan Y, Eric CH, Stephanie HY. Associating liver partition and portal vein ligation for staged hepatectomy: the current role and development. Hepatobiliary Pancreat Dis Int. 2017;16(1):17-26.

2. Clavien PA, Petrowsky H, DeOliveira ML, Graf R. Strategies for safer liver surgery and partial liver transplantation. N Engl J Med 2007; 356:1545-1559.

3. Hernandez-Alejandro R, Bertens KA, PinedaSolis K, Croome KP. Can we improve the morbidity and mortality associated with the associating liver partition with portal vein ligation for staged hepatectomy (ALPPS) procedure in the management of colorectal liver metastases? Surgery 2015;157:194-201.

4. Røsok BI, Björnsson B, Sparrelid E, Hasselgren K, Pomianows- ka $E$, Gasslander T, et al. Scandinavian multicenter study on the safety and feasibility of the associating liver partition and portal vein ligation for staged hepatectomy procedure. Surgery 2016;159:12791286.

5. Wan L. Eric CH. Modifications of ALPPS-from complex to more complex or from complex to less complex operations. Hepatobiliary Pancreat Dis Int, 2017;16(4):346-352. 